\title{
Array-based comparative genomic hybridization is more informative than conventional karyotyping and fluorescence in situ hybridization in the analysis of first-trimester spontaneous abortion
}

Jinsong Gao', Congcong Liu², Fengxia Yao², Na Hao², Jing Zhou², Qian Zhou², Liang Zhang ${ }^{3}$, Xinyan Liu', Xuming Bian ${ }^{1}$ and Juntao Liu $^{1 *}$

\begin{abstract}
Background: Array-based comparative genomic hybridization $(\mathrm{aCGH})$ is a new technique for detecting submicroscopic deletions and duplications, and can overcome many of the limitations associated with classic cytogenetic analysis. However, its clinical use in spontaneous abortion needs comprehensive evaluation. We used aCGH to investigate chromosomal imbalances in 100 spontaneous abortions and compared the results with Gbanding karyotyping and fluorescence in situ hybridization (FISH). Inconsistent results were verified by quantitative fluorescence PCR.

Results: Abnormalities were detected in 61 cases. aCGH achieved the highest detection rate $(93.4 \%, 57 / 61)$ compared with traditional karyotyping $(77 \%, 47 / 61)$ and FISH analysis $(68.9 \%, 42 / 61)$. aCGH identified all chromosome abnormalities reported by traditional karyotyping and interphase FISH analysis, with the exception of four triploids. It also detected three additional aneuploidy cases in 37 specimens with 'normal' karyotypes, one mosaicism and 10 abnormalities in 14 specimens that failed to grow in vitro.

Conclusions: $\mathrm{aCGH}$ analysis circumvents many limitations in traditional karyotyping or FISH. The accuracy and efficiency of aCGH in spontaneous abortions highlights its clinical usefulness for the future. As aborted tissues have the potential to be contaminated with maternal cells, the threshold value of detection in aCGH should be lowered to avoid false negatives.
\end{abstract}

Keywords: Spontaneous abortion, Aneuploidy, Karyotyping, Array-based comparative genomic hybridization

\section{Background}

Spontaneous abortion is a common clinical problem that affects $10-15 \%$ of all clinically recognized human pregnancies, and mostly occurs in the first trimester [1]. Unbalanced chromosomal abnormalities account for $50-60 \%$ of fetal loss during this period, based on the results of cytogenetic studies [2] that provide valuable

\footnotetext{
* Correspondence: Liu_jt@sohu.com

'Department of Obstetrics and Gynecology, Peking Union Medical College Hospital, Chinese Academy of Medical Science, Shuai Fu Yuan No.1, Dongdan, Beijing 100730, People's Republic of China

Full list of author information is available at the end of the article
}

insights into the possible genetic causes of miscarriage and can determine recurrent risks.

Classic cytogenetic analysis is often the only genetic laboratory evaluation performed for spontaneous abortions. However, it has many limitations in the analysis of miscarriage. It relies on the successful culture of fetal tissue and preparation of metaphase cells, yet the successful rate of conventional karyotyping of miscarriage tissue is relatively low, ranging from 60 to $90 \%$ because of the in vivo death of tissue associated with spontaneous abortion, technical problems with culture growth or poor chromosome morphology [3-5]. Moreover, the information it provides 
is limited to numerical chromosomal abnormalities and gross structural rearrangements at a resolution of 5$10 \mathrm{Mb}$ [6]. It has also been suggested that classic cytogenetics of spontaneous abortion might yield a false-positive normal karyotype or selected abnormal karyotype that permits cell proliferation in vitro [7]. In addition, standard cytogenetic diagnosis is a labor-intensive procedure, requiring the short- or long-term culture of fetal tissue.

Other rapid molecular cytogenetic techniques such as fluorescence in situ hybridization (FISH), quantitative fluorescence polymerase chain reaction (QF-PCR) and subtelomeric multiplex ligation-dependent probe amplification, which do not require cell culture, avoid some of these karyotyping pitfalls $[8,9]$. However, although these techniques detect the majority of chromosomal aberrations in spontaneous miscarriages, they use probes and primers that only target a selection of chromosomes or specific subtelomeric loci, thereby missing information about the remaining genome [10-12].

Array-based comparative genomic hybridization $(\mathrm{aCGH})$ is a powerful new molecular cytogenetic technique that enables the genome-wide analysis of DNA copy numbers. It allows the simultaneous screening of gains and losses at thousands of targets while offering the advantages of high resolution and high throughput [13]. Many of the limitations of routine G-banding analysis, including cell culture failure and poor chromosome morphology, are circumvented by the use of genomic DNA. Unbalanced gains or losses of genetic material across the genome including those invisible to Gbanding analysis can also be detected by aCGH [14-16].

When aCGH techniques are employed in the postnatal and prenatal population, there appears to be an increased detection rate of chromosomal imbalances, compared with conventional karyotyping $[14,17,18]$. Recently, aCGH has been considered a particularly useful alternative to conventional karyotyping in the field of diagnosis. However, although it is rapidly becoming the primary tool for the postnatal genetic evaluation of neonates and infants with dysmorphic features or cognitive difficulties, its use in routine prenatal diagnosis and miscarriage evaluation is still being investigated.

Thus far, the application of aCGH in miscarriages has been limited to no more than 500 cases worldwide. To our knowledge, seven studies have evaluated aCGH in the analysis of spontaneous abortion specimens $[7,19$ 24]. aCGH appears to have an increased detection rate of chromosomal abnormality compared with conventional karyotyping. It was capable of detecting additional abnormalities in about $10 \%$ cases with normal karyotype mainly due to maternal cell contamination or submicroscopic chromosomal changes, and nearly $50 \%$ abnormalities in samples with culture failure when karyotyping was impossible. As it has also been suggested that lethal submicroscopic chromosomal changes can cause miscarriages [25,26], aCGH could become a complementary or even alternative method to traditional cytogenetic technique.

There are nearly 20,000,000 neonates each year in China with $10-15 \%$ ending in miscarriages. However, the genetic analysis of spontaneous abortions is seldom carried out because of limited cytogenetic resources. For this reason, the application of aCGH in spontaneous abortion analysis has never been reported in Chinese populations, yet it needs further evaluation for its clinical use in the detection of genomic imbalances in the cytogenetic evaluation of spontaneous miscarriages.

In this study, therefore, 100 spontaneous abortion specimens analyzed by G-banding and multiplex FISH were tested by aCGH arrays to evaluate the accuracy and efficiency of aCGH in the analysis of first trimester spontaneous abortions.

\section{Results}

Karyotyping failed in 14 (14\%) samples as a result of culture failure, while aCGH and FISH analyses were successful in all cases. Overall, chromosome abnormalities were detected in 61 (61\%) of the 100 spontaneous abortion specimens. Culturing detected abnormalities in 47 of all specimens (54.6\% of those successfully karyotyped, and $77 \%$ of all abnormalities). FISH analysis using three probe sets targeting chromosomes 13, 16, 18, 21, 22, X and $\mathrm{Y}$ detected 42 abnormalities (accounting for $68.9 \%$ of all abnormalities). The remaining 19 chromosomal aberrations (31.1\%) involving 11 different chromosomes were not detected by FISH because of probe limitations. aCGH detected chromosomal aberrations in 57 samples (93.4\% of all abnormalities) but missed four triploid cases. The chromosome distribution of abnormalities is shown in Figure 1.

In accordance with previous reports, autosomal trisomy was predominant, accounting for $80.3 \%$ (49/61) of all chromosome abnormalities. Trisomy $16(\mathrm{n}=16)$ and $22(\mathrm{n}=10)$ were the most frequently encountered abnormalities, while trisomy $21(\mathrm{n}=4), 45, \mathrm{X}(\mathrm{n}=4)$ and triploid $(n=4)$ were the joint second most common abnormalities. These account for $62.3 \%(38 / 61)$ of all abnormalities.

A comparison of conventional cytogenetic, FISH and aCGH analyses is shown in Table 1 . Seventy-five cases (87.2\% of those successfully karyotyped) analyzed by aCGH were in exact concordance with the karyotype results. Of these matches, 32 showed a normal karyotype and 43 showed abnormal results in both tests, including 39 cases with autosomal trisomy, two cases with sex chromosome abnormalities (monosomy $\mathrm{X}$ ), one case with monosomy 21 and one case with an 18p deletion. 


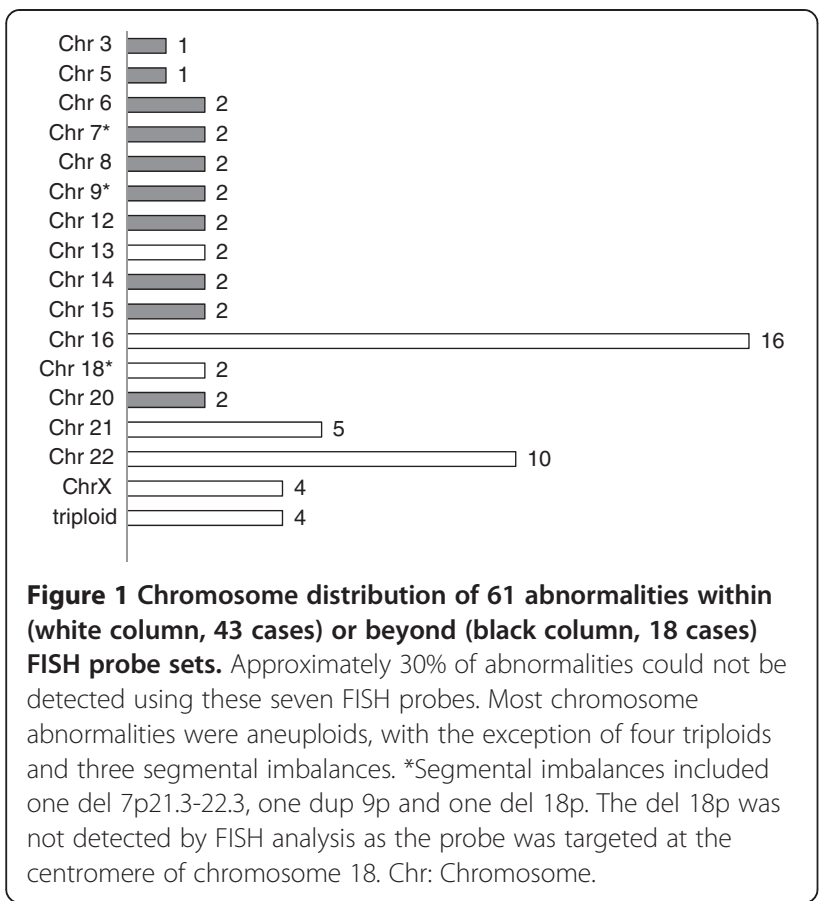

Discrepancies between aCGH and cytogenetic results occurred in $11(12.8 \%)$ of 86 karyotyped cases (Table 1$)$. Three 46, XX karyotypes (cases 12-14) were proved to be a maternal overgrowth of fetal cells, with one (case 14) diagnosed as a trisomy 5 male by aCGH analysis. Two 46, XY karyotypes were diagnosed as trisomy 21 (case 36) and trisomy 14 (case 37) by aCGH. One trisomy 22 shown by karyotype and FISH analysis (case 74) was suggested instead to be a mosaicism for trisomy 22 and trisomy 9 by aCGH analysis. The additional chromosome abnormalities detected by aCGH were all verified by FISH analysis or QF-PCR (Figure 2). Three triploids diagnosed by karyotype and FISH analysis (cases $82-84$ ) were all found to be normal by aCGH. Two tetraploid cases (85 and 86 ) were proved to be culture artifacts. One trisomy 16 was confused with trisomy 20 because of poor chromosome morphology. It was later confirmed to be trisomy 16 by aCGH.

In 14 specimens that failed to yield a karyotype, 11 (78.6\%) showed abnormalities as detected by either FISH or aCGH. aCGH results were then compared with FISH analysis (Table 2). FISH analysis verified six aCGH results but missed one trisomy 8 , one trisomy 15 , one $9 p$ duplication and one $7 \mathrm{p}$ deletion (del 7p21.3-p22.3,$11.72 \mathrm{Mb}$ ), which were not identifiable by the probe sets. One normal sample in aCGH was proven to be triploid by FISH analysis.

Out of 61 abnormal specimens, we detected seven samples with a high maternal cell contamination (MCC) rate by reducing the threshold value of fold change in aCGH. Table 3 gives the MCC rates of these specimens.
Table 1 Comparison of karyotyping, FISH and aCGH analysis $(n=86)$

\begin{tabular}{|c|c|c|c|}
\hline Case No & Karyotype results & FISH results & Array CGH results ${ }^{\mathrm{e}}$ \\
\hline $1-11$ & $46, X X$ & $X X$ & Normal \\
\hline $12-13^{a}$ & $46, X X$ & $X Y$ & Normal \\
\hline $14^{\mathrm{a}}$ & $46, X X$ & $X Y$ & $+5^{b}$ \\
\hline $15-35$ & $46, X Y$ & $X Y$ & Normal \\
\hline 36 & $46, X Y$ & $X Y,+21$ & +21 \\
\hline 37 & $46, X Y$ & $X Y$ & $+14^{\mathrm{b}}$ \\
\hline 38 & $47, X X,+3$ & $X X$ & +3 \\
\hline 39 & $47, X X,+6$ & $X X$ & +6 \\
\hline 40 & $47, X Y,+6$ & $X Y$ & +6 \\
\hline 41 & $47, X X,+7$ & $X X$ & +7 \\
\hline 42 & $47, X X,+8$ & $X X$ & +8 \\
\hline 43 & $47, X X,+9$ & $X X$ & +9 \\
\hline $44-45$ & $47, X Y,+12$ & $X Y$ & +12 \\
\hline 46 & $47, X X,+13$ & $X X,+13$ & +13 \\
\hline 47 & $47, X X,+14$ & $X X$ & +14 \\
\hline 48 & $47, X X,+15$ & $X X$ & +15 \\
\hline $49-53$ & $47, X X,+16$ & $X X,+16$ & +16 \\
\hline $54-62$ & $47, X Y,+16$ & $X Y,+16$ & +16 \\
\hline 63 & $47, X Y,+16$ & $X Y,+16 / X Y$ mosaic & +16 \\
\hline 64 & $47, X X,+18$ & $X X,+18$ & +18 \\
\hline $65-66$ & $47, X X,+20$ & $X X$ & +20 \\
\hline $67-68$ & $47, X X,+21$ & $X X,+21$ & +21 \\
\hline 69 & $47, X Y,+21$ & $X Y,+21$ & +21 \\
\hline $70-72$ & $47, X X,+22$ & $X X,+22$ & +22 \\
\hline 73 & $47, X X,+22$ & $X X,+22 / X X$ mosaic & +22 \\
\hline 74 & $47, X X,+22$ & $x X_{1}+22$ & $+9^{b},+22$ \\
\hline $75-77$ & $47, X Y,+22$ & $X Y,+22$ & +22 \\
\hline 78 & $46, X Y$, del $18 p$ & $X Y$ & del $18 p$ \\
\hline 79 & $45, X Y,-21$ & $X Y,-21$ & -21 \\
\hline $80-81$ & $45, x$ & Monosomy X & Monosomy X \\
\hline $82-84$ & Triploid & Triploid & Normal \\
\hline $85-86$ & Tetraploid $^{\mathrm{c}}$ & Normal & Normal \\
\hline All abnormal & $47^{d}$ & 35 & 47 \\
\hline
\end{tabular}

anly maternal cell cultured.

berified by QF-PCR.

'due to a culture artifact $(92, \mathrm{XXXX}$ and $92, \mathrm{XXYY})$.

dexcluding two culture artifact tetraploids.

eThe sex chromosome in aCGH was in accordance with FISH analysis.

\section{Discussion}

In this study, aCGH was compared with traditional karyotyping and interphase FISH analysis for the detection of chromosomal abnormalities in spontaneous abortions. Overall, aCGH showed the highest detection rate $(93.4 \%$, 57/61) compared with karyotyping $(77 \%, 47 / 61)$ and FISH analysis $(68.9 \%, 42 / 61)$. Our study demonstrates that aCGH analysis of spontaneous abortions is both accurate 


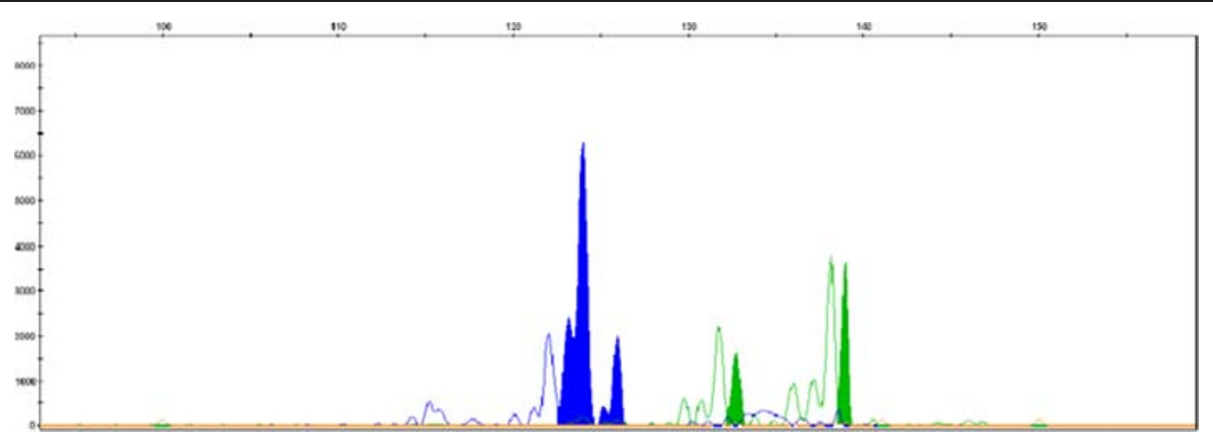

Figure 2 Electrophoretograms of QF-PCR amplifications in case 37. The $x$-axis shows the length of the PCR products in base pairs and the $y$ axis shows the fluorescence intensity in arbitrary units. DNA samples were amplified with two markers for chromosome 14: D14S985 (blue) and D14S1007 (green). Both markers are informative and show two peaks with a 2:1 ratio, confirming the result of trisomy 14 in array CGH.

and efficient, which augurs well for its clinical usefulness. To our knowledge, this is the first such study to be reported of the Chinese population.

The distribution of chromosome abnormalities identified in our study was similar to other reports $[6,12]$ (see Figure 1). However, submicroscopic imbalances were less prevalent, most likely because they were not lethal. We identified one case with a single copy loss of $11.72 \mathrm{Mb}$ in the 7p21.3-p22.3 region. Such deletions have previously been found in postnatal patients with mental retardation or development delay [27], suggesting that the spontaneous abortions caused by this microdeletion must be occasional even if they are considered pathogenic.

In another case, the array data suggested mosaicism for trisomy 9 and 22, which was not identified by cytogenetic analysis. It is conceivable that the cell line with trisomy 9 is slow to grow and divide, thereby presenting

Table 2 Comparison of FISH and aCGH analysis of cases without karyotype results $(n=14)$

\begin{tabular}{lll}
\hline Case No. & FISH & aCGH $^{\mathbf{a}}$ \\
\hline 87 & $\mathrm{XX}$ & Normal \\
88 & $\mathrm{XY}$ & Normal \\
89 & $\mathrm{XY}$ & Normal \\
90 & $\mathrm{XX}$ & del 7p21.3-p22.3 (-11.72 Mb) \\
91 & $\mathrm{XX}$ & +8 \\
92 & $\mathrm{XX}$ & dup 9p \\
93 & $\mathrm{XX}$ & +15 \\
94 & $\mathrm{XY},+13$ & +13 \\
95 & $\mathrm{XY},+16$ & +16 \\
96 & $\mathrm{XX},+22$ & +22 \\
97 & $\mathrm{XY},+22$ & +22 \\
98 & Monosomy $X$ & Monosomy $X$ \\
99 & Monosomy $X$ & Monosomy $X$ \\
100 & Triploid & Normal \\
All abnormal & 7 & 10 \\
\hline
\end{tabular}

${ }^{\mathrm{a}}$ The sex chromosome in aCGH was in accordance with FISH analysis. very few mitotic cells. This example highlights the limitation of cytogenetic analysis which relies on cell culture and demonstrates the potential of aCGH to detect the presence of mosaicism [22].

Karyotyping inaccuracies have been shown to be a result of maternal cell overgrowth or selected karyotypes that permit cell proliferation in vitro [7]. The culture failure rate and $\mathrm{MCC}$ rate of our study were similar to those of other reports $[3,17,28]$, although the cytogenetic factors of cell death in vitro have not been thoroughly investigated. It is possible that cell culture failure is a marker of particular genomic imbalances incompatible with normal cell proliferation. As shown in our results, abnormalities were more frequently detected in specimens with culture failure. If this hypothesis is true, then the standard cytogenetic analysis of spontaneous abortions may underestimate the frequency and diversity of detected chromosomal abnormalities.

Insufficient enumeration probes could cause inaccuracies in FISH analysis. As shown in Figure 1, the distribution of aberration was scattered throughout most chromosome types. Although approximately $70 \%$ chromosome abnormalities could be detected by this multiplex interphase FISH, consistent with other reports [12], more than $30 \%$ of abnormalities could not be detected. As the use of probes

Table 3 Abnormal samples with high MCC rate detected by lower threshold value of aCGH

\begin{tabular}{|c|c|c|c|}
\hline Case No. & $\begin{array}{l}\text { Routine threshold } \\
\text { value } \log _{2} \text { ratio }=0.38\end{array}$ & $\begin{array}{l}\text { Lowered threshold } \\
\text { valueLog } 2 \text { ratio }=0.26\end{array}$ & $\begin{array}{l}\text { MCC } \\
\text { rate (\%) }\end{array}$ \\
\hline 40 & Normal & +6 & 17.1 \\
\hline 41 & Normal & +7 & 30.8 \\
\hline 53 & Normal & +16 & 29.2 \\
\hline 59 & Normal & +16 & 22.3 \\
\hline 60 & Normal & +16 & 20.6 \\
\hline 61 & Normal & +16 & 18.8 \\
\hline 62 & Normal & +16 & 22.3 \\
\hline
\end{tabular}

MCC: maternal cell contamination. 
that target all chromosomes would be expensive and unrealistic, this limits the application of FISH in the complete analysis of spontaneous abortion.

Aborted tissue is a mixture of conceptus, chorionic villi and deciduas, so is likely to be contaminated with maternal cells. It is therefore essential to carefully detach the deciduas from the chorionic villi as much as possible to decrease the MCC rate, although this is difficult to achieve. To overcome this problem, we reduced the detection threshold to a 1.2 -fold change ( $0.26 \log _{2}$ ratio) to see if this would increase the detection rate without increasing the false positive rate. Under this threshold value, we detected seven additional chromosomal aberrations that had originally showed a negative result under aCGH routine protocols. Thus it seems reasonable to adjust the threshold value to $0.26 \log _{2}$ ratio fold change when analyzing spontaneous abortion specimens with aCGH. Although all deviations from the normal range were considered to be MCC, placenta mosaicisms would also meet this analysis criterion. However, distinguishing between these two situations has no obvious clinical significances. In addition, different microarray platforms have their own system noises. Since we deduce the $\mathrm{W}$ value and calculate the MCC rate (or mosaic rate) on an Agilent 60-mer Oligo aCGH platform (see Methods), it is important that the $\mathrm{W}$ value and its standard error in this algorithm be Agilent platform-specific.

A limitation of aCGH in the analysis of spontaneous abortion is that it cannot reliably detect polyploidy, which accounts for about $2-10 \%$ of all spontaneous abortions $[10,28]$. This is mainly because of the global normalization used in microarray-based methods. Although a previous study overcame these problems by using $47, \mathrm{XXY}$ cells as control DNA to enable the detection of some triploids [29], this was not conducted in the present study but remains a possibility for future work.

\section{Conclusions}

The present study demonstrates that the DNA-based aCGH technology overcomes many limitations of routine cytogenetic analysis used in the analysis of spontaneous abortion specimens while enhancing the detection rate of chromosome aberrations. Although its current costs are relatively expensive, we expect the price to decrease in the near future with increases in the number of arrays consumed in clinical applications. At that time, aCGH may become a cost-effective method in the analysis of spontaneous abortion and other chromosome abnormality diagnosis.

\section{Methods}

\section{Specimen preparation}

A total of 100 samples were received from women who experienced spontaneous miscarriage before 12 weeks of gestation at Peking Union Medical College Hospital, Beijing, China. All patients gave informed consent for participation in the research. The mean maternal age of the patient group was 32 years (range, 24-45 years); all were primigravidae, although there had been a previous abortion in two cases.

After termination of the pregnancies, a small portion of chorionic villi samples (CVS) was placed in a sterile container with $0.9 \%$ normal saline and sent to the laboratory within 24 hours. The specimen was examined grossly, washed clean of blood and maternal deciduas and divided into three parts for cell culture, preparation of noncultured single-cell suspensions and DNA extraction.

\section{Karyotype analysis}

Approximately 20-30 mg CVS tissue was used for cell culture. Following collagenase and trypsin dissociation of the specimen, two primary cultures from the tissue samples were established using standard methods. Cultures were harvested after approximately 1-2 weeks. Karyotyping was performed on G-banded chromosome preparations. A minimum of five, but preferentially 20 , cells were analyzed per sample.

\section{FISH analysis}

About $5 \mathrm{mg}$ of tissues was used for FISH analysis. Noncultured single-cell suspensions were prepared by disaggregation in 60\% acetic acid, then fixed and stored in 3:1 methanol/acetic acid.

Based on knowledge about the frequencies of specific trisomies in spontaneous abortions and the availability of commercial multiplex probe sets, FISH was performed using three multicolor probe mixtures (GP Medical Technologies Inc., Beijing, China). Probe mix 1 and mix 2 contain locus-specific probes that identify chromosomes 13, 21 and 16, 22 respectively. Probe mix 3 contains centromere site probes that identify chromosomes 18, X and Y. Each probe set was applied to one of the two slides from each case. The probes were directlabeled with different fluorophores, which could be visualized with appropriate filter combinations. Hybridization and post-washing conditions were performed according to the manufacturer's instructions. Slides were counterstained with $15 \mathrm{ml}$ of a very dilute 4,6-diamidino-2-phenylindole antifade solution for 10 minutes and observed under a fluorescent microscope (BX51, Olympus, Tokyo, Japan) equipped with an appropriate filter.

Nuclear signals were scored using a $\times 100$ oil objective. Fifty nuclei were scored for each of the seven chromosomes. The cut-off rates scheme was prepared according to a previous report [30] and our own experience. In normal diploid cells, two signals were observed for each 
chromosome from a mean of $90 \%$ of scored cells. Aneuploidy was diagnosed when $>60 \%$ of cells showed an abnormal number of signals. More nuclei $(\geq 100)$ were scored if $10-60 \%$ of cells showed abnormalities. A value between 10 and 20\% was regarded as unclear but likely to be normal. A value $>20 \%$ was regarded as mosaicism or suspected aneuploidy.

\section{aCGH analysis}

aCGH analysis was used to examine all 100 spontaneous abortion samples. Total DNA was extracted from uncultured CVS tissues with a commercially available Genomic DNA Extraction Kit (BioChain Institute Inc., Newark, CA) according to the manufacturer's instructions. For each aCGH experiment, $400 \mathrm{ng}$ each of purified DNA and normal sex-matched DNA (BioChain Institute) was digested with $10 \mathrm{U}$ Alu I and $10 \mathrm{U}$ Rsa I (Promega, Madison, WI) and differentially labeled with cyanine-5 (cy5) and cyanine-3 (Cy3) fluorescent dyes using a Genomic DNA Enzymatic Labeling Kit (Agilent, Santa Clara, CA). aCGH analysis was performed using $8 \times 60 \mathrm{~K}$ commercial arrays (Agilent). This platform contains 60-mer oligonucleotide probes spanning the entire human genome with an overall mean probe spacing of $50 \mathrm{~kb}$. After hybridization, the arrays were scanned using a dual-laser scanner (Agilent) and the images were extracted and analyzed using Feature Extraction software (Agilent) and Workbench genomics software, respectively.

\section{MCC analysis}

An aborted conceptus is likely to be contaminated with maternal cells, resulting in the failure of karyotyping analysis. In aCGH analysis, DNA was directly extracted from CVS tissues so might be expected to contain maternal DNA. If MCC-containing CVS samples are analyzed according to unitary samples, false negative CVS DNA abnormalities may be revealed. We therefore adopted a mixing algorithm to detect the chromosome abnormalities in CVS tissues. Since trisomies account for most spontaneous abortions, we used trisomy 21 as an example to establish the mixing model. Thus, in a trisomy sample contaminated with maternal cells, the measured ratio is $M=R+(1-R)^{*} T$. This algorithm has been used in the aCGH analysis of tumor tissues, which often contain normal cells [31]. Here, R is the MCC proportion, and $\mathrm{T}$ is the ratio of trisomy DNA to normal control, which should theoretically equal 1.5 , inferring that $M=1.5-0.5 R$. The $M$ value is linear to $R$. When $R$ equals zero, representing no contamination, the $M$ value will be 1.5; when $\mathrm{R}$ equals 1.0 , meaning that all cells derive from the mother, the $M$ value will also be 1.0.

To detect chromosome abnormalities, a threshold value should be determined considering the true biological change and the system noise. Agilent aCGH standard protocols set the threshold value as a 1.3-fold change for one copy number amplification or loss, which corresponds to a $\log _{2}$ ratio $<-0.38$ or $>0.38$. When considering MCC in miscarriage tissues, we established an equation $\mathrm{M}=\mathrm{Mc}+\mathrm{W}$, where $\mathrm{Mc}$ denotes the threshold value of fold change that optimally detects trisomy. Above the $M_{c}$ value no abnormality is detected, while below the $M_{c}$, the abnormality is reported. W is a constant reflecting precision of the aCGH platform that can be estimated by comparing the non-contaminated trisomy samples with normal samples. We employed 10 trisomy 21 syndrome samples from peripheral blood and calculated $W=0.11 \pm 0.04$. Deducing from $M=M c+W$ for $\mathrm{Mc}=1.39$, this suggests that the trisomy DNA is not contaminated with normal DNA, which agrees with a previous comparison between three and two copies of the X chromosome [32].

To detect chromosome abnormalities in miscarriages with potential MCC, we reduced the detection threshold to a 1.2 -fold change $\left(0.26 \log _{2}\right.$ ratio) with at least 10 consecutive probes. Using this threshold value, the MCC rate was $32.5 \%$. Reducing the threshold still further could lead to the emergence of false positives because of platform system noise (data not shown).

\section{QF-PCR}

QF-PCR of DNA was performed to verify the results of aCGH if these revealed unbalanced chromosomal abnormalities that did not correlate with the karyotype. By amplifying highly polymorphic regions of short tandem repeats (STR) specific for a particular chromosome, we could detect dosage ratios of the PCR products by analyzing the fluorescent peak areas shown by a Genetic Analyzer. In normal heterozygotes, the ratio of fluorescent activity for the two peaks corresponding to the PCR products should be within the range $0.8-1.4$ (disomic diallelic). In a trisomic specimen, the three doses of an STR marker can be detected either as three peaks of fluorescent activities with a 1:1:1 ratio (trisomic triallelic) or as a pattern of two peaks with a 1:2 ratio (trisomic diallelic) [33].

\section{Competing interest statement}

The authors declare that they have no competing interests.

\section{Authors' contributions}

JG drafted the manuscript, performed array CGH and carried out data analysis. CL performed FISH. FY performed QF-PCR. NH and JZ performed karyotyping. QZ and LZ performed array CGH and participated in the study coordination. XL performed clinical evaluations of the pregnancies and participated in the coordination of the project. XB performed clinical evaluations of the pregnancies and helped to draft the manuscript. JL conceived the study, participated in its design and coordination and also approved the manuscript. All authors have read and approved the manuscript.

\section{Acknowledgments}

Our study was funded by Chinese Medical Association (Special Fund of Chinese Medical Association, CAMB012010). We are also grateful to other laboratory members for their enthusiastic participation. 


\section{Author details}

'Department of Obstetrics and Gynecology, Peking Union Medical College Hospital, Chinese Academy of Medical Science, Shuai Fu Yuan No.1, Dongdan, Beijing 100730, People's Republic of China. ${ }^{2}$ Department of Molecular Genetics \& Cytogenetics, Peking Union Medical College Hospital, Chinese Academy of Medical Science, Shuai Fu Yuan No.1, Dongdan, Beijing 100730, People's Republic of China. ${ }^{3}$ BioChain (Beijing) Science and Technology Inc., No.7A, Yongchang North Rd, Beijing Economictechnological Development Area, Beijing 100176, People's Republic of China.

Received: 12 April 2012 Accepted: 20 May 2012

Published: 16 July 2012

\section{References}

1. Zinaman MJ, Clegg ED, Brown CC, O'Connor J, Selevan SG: Estimates of human fertility and pregnancy loss. Fertil Steril 1996, 65:503-509.

2. Byrne $\lrcorner,$, Ward K: Genetic factors in recurrent abortion. Clin Obstet Gynecol 1994, 37:693-704.

3. Nagaishi MYT, linuma K, Shimomura K, Berend SA, Knops J: Chromosome abnormalities identified in 347 spontaneous abortions collected in Japan. J Obstet Gynaecol Res 2004, 30:237-241.

4. Lin CC, De Braekeleer M, Jamro H: Cytogenetic studies in spontaneous abortion: the Calgary experience. Can J Genet Cytol 1985, 27:565-570.

5. Greenwold N, Jauniaux E: Collection of villous tissue under ultrasound guidance to improve the cytogenetic study of early pregnancy failure. Hum Reprod 2002, 17:452-456.

6. Gardner MR, Sutherland GR: Cytogenetics of spontaneous abortions and later pregnancy loss. In Chromosome abnormalities and genetic counseling. 3rd edition. Edited by Gardner MR, Sutherland GR. New York: Oxford University Press; 2004:343-345.

7. Benkhalifa M, Kasakyan S, Clement P, Baldi M, Tachdjian G, Demirol A, Gurgan T, Fiorentino F, Mohammed M, Qumsiyeh MB: Array comparative genomic hybridization profiling of first-trimester spontaneous abortions that fail to growin vitro. Prenat Diagn 2005, 25:894-900.

8. Bryndorf T, Christensen B, Vad M, Parner J, Brocks V, Philip J: Prenatal detection of chromosome aneuploidies by fluorescence in situ hybridization: experience with 2000 uncultured amniotic fluid samples in a prospective preclinical trial. Prenat Diagn 1997, 17:333-341.

9. Bryndorf T, Christensen B, Vad M, Parner J, Carelli MP, Ward BE, Klinger KW, Bang J, Philip J: Prenatal detection of chromosome aneuploidies in uncultured chorionic villus samples by FISH. Am J Hum Genet 1996, 59:918-926.

10. Bruno DL, Burgess $T$, Ren $H$, Nouri S, Pertile MD, Francis DI, Norris F, Kenney BK, Schouten J, Andy Choo KH, et al: High-throughput analysis of chromosome abnormality in spontaneous miscarriage using an MLPA subtelomere assay with an ancillary FISH test for polyploidy. Am J Med Genet A 2006, 140:2786-2793

11. Diego-Alvarez D, Garcia-Hoyos M, Trujillo MJ, Gonzalez-Gonzalez C, Rodriguez de Alba M, Ayuso C, Ramos-Corrales C, Lorda-Sanchez I: Application of quantitative fluorescent PCR with short tandem repeat markers to the study of aneuploidies in spontaneous miscarriages. Hum Reprod 2005, 20:1235-1243.

12. Jobanputra V, Sobrino A, Kinney A, Kline J, Warburton D: Multiplex interphase FISH as a screen for common aneuploidies in spontaneous abortions. Hum Reprod 2002, 17:1166-1170.

13. Salman M, Jhanwar SC, Ostrer H: Will the new cytogenetics replace the old cytogenetics? Clin Genet 2004, 66:265-275

14. Hillman SC, Pretlove S, Coomarasamy A, McMullan DJ, Davison EV, Maher ER, Kilby MD: Additional information from array comparative genomic hybridization technology over conventional karyotyping in prenatal diagnosis: a systematic review and meta-analysis. Ultrasound Obstet Gynecol 2011, 37:6-14

15. Pinkel D, Segraves R, Sudar D, Clark S, Poole I, Kowbel D, Collins C, Kuo WL, Chen $C$, Zhai $Y$, et al: High resolution analysis of DNA copy number variation using comparative genomic hybridization to microarrays. Nat Genet 1998, 20:207-211.

16. Snijders AM, Nowak N, Segraves R, Blackwood S, Brown N, Conroy J, Hamilton G, Hindle AK, Huey B, Kimura K, et al: Assembly of microarrays for genome-wide measurement of DNA copy number. Nat Genet 2001, 29:263-264
17. Lee CN, Lin SY, Lin CH, Shih JC, Lin TH, Su YN: Clinical utility of array comparative genomic hybridisation for prenatal diagnosis: a cohort study of 3171 pregnancies. BJOG 2012, 119:614-625

18. Miller DT, Adam MP, Aradhya S, Biesecker LG, Brothman AR, Carter NP, Church DM, Crolla JA, Eichler EE, Epstein CJ, et al: Consensus statement: chromosomal microarray is a first-tier clinical diagnostic test for individuals with developmental disabilities or congenital anomalies. Am J Hum Genet 2010, 86:749-764.

19. Borovik CL PA, da Silva L, Krepischi-Santos A, Costa S, Rosenberg C: Array-CGH testing in spontaneous abortions with normal karyotypes. Genet Mol Bio 2008, 31:416-422.

20. Menten B, Swerts K, Chiaie B, Janssens S, Buysse K, Philippé J, Speleman F: Array comparative genomic hybridization and flow cytometry analysis of spontaneous abortions and mors in utero samples. BMC Med Genet 2009, 10:89-93.

21. Robberecht C, Schuddinck V, Fryns JP, Vermeesch JR: Diagnosis of miscarriages by molecular karyotyping: benefits and pitfalls. Genet Med 2009, 11:646-654

22. Schaeffer AJ, Chung J, Heretis K, Wong A, Ledbetter DH, Lese Martin C: Comparative genomic hybridization-array analysis enhances the detection of aneuploidies and submicroscopic imbalances in spontaneous miscarriages. Am J Hum Genet 2004, 74:1168-1174.

23. Shimokawa O, Harada N, Miyake N, Satoh K, Mizuguchi T, Niikawa N, Matsumoto N: Array comparative genomic hybridization analysis in firsttrimester spontaneous abortions with 'normal' karyotypes. Am J Med Genet A 2006, 140:1931-1935.

24. Zhang YX, Zhang YP, Gu Y, Guan FJ, Li SL, Xie JS, Shen Y, Wu BL, Ju W, Jenkins EC, et al: Genetic analysis of first-trimester miscarriages with a combination of cytogenetic karyotyping, microsatellite genotyping and arrayCGH. Clin Genet 2009, 75:133-140.

25. Rajcan-Separovic E, Diego-Alvarez D, Robinson WP, Tyson C, Qiao Y, Harvard C, Fawcett C, Kalousek D, Philipp T, Somerville MJ, et al: Identification of copy number variants in miscarriages from couples with idiopathic recurrent pregnancy loss. Hum Reprod 2010, 25:2913-2922.

26. Rajcan-Separovic E, Qiao Y, Tyson C, Harvard C, Fawcett C, Kalousek D, Stephenson M, Philipp T: Genomic changes detected by array CGH in human embryos with developmental defects. Mol Hum Reprod 2010, 16:125-134.

27. Xiang B, Zhu H, Shen Y, Miller DT, Lu K, Hu X, Andersson HC, Narumanchi TM, Wang Y, Martinez JE, et al: Genome-wide oligonucleotide array comparative genomic hybridization for etiological diagnosis of mental retardation: a multicenter experience of 1499 clinical cases. J Mol Diagn 2010, 12:204-212.

28. Lomax B, Tang S, Separovic E, Phillips D, Hillard E, Thomson T, Kalousek DK: Comparative genomic hybridization in combination with flow cytometry improves results of cytogenetic analysis of spontaneous abortions. Am J Hum Genet 2000, 66:1516-1521.

29. Ballif BC, Kashork CD, Saleki R, Rorem E, Sundin K, Bejjani BA, Shaffer LG: Detecting sex chromosome anomalies and common triploidies in products of conception by array-based comparative genomic hybridization. Prenat Diagn 2006, 26:333-339.

30. Liehr T: Rapid Prenatal Diagnostics in the Interphase Nucleus: Procedure and Cut-off Rates. J Histochem Cytochem 2005, 53:289-291.

31. van de Wiel MA, Picard F, van Wieringen WN, Ylstra B: Preprocessing and downstream analysis of microarray DNA copy number profiles. Brief Bioinform 2011, 12:10-21.

32. Pollack JR, Perou CM, Alizadeh AA, Eisen MB, Pergamenschikov A, Williams CF, Jeffrey SS, Botstein D, Brown PO: Genome-wide analysis of DNA copy-number changes using cDNA microarrays. Nat Genet 1999, 23:41-46.

33. Hulten MA, Dhanjal S, Pertl B: Rapid and simple prenatal diagnosis of common chromosome disorders: advantages and disadvantages of the molecular methods FISH and QF-PCR. Reproduction 2003, 126:279-297.

doi:10.1186/1755-8166-5-33

Cite this article as: Gao et al:: Array-based comparative genomic hybridization is more informative than conventional karyotyping and fluorescence in situ hybridization in the analysis of first-trimester spontaneous abortion. Molecular Cytogenetics 2012 5:33. 